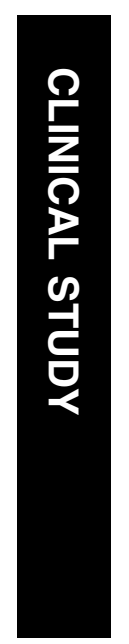

\title{
Influence of age, central corneal thickness, and quality score on dynamic contour tonometry
}

${ }^{1}$ Department of Ophthalmology; State University of Campinas (UNICAMP), Campinas, SP, Brazil

${ }^{2}$ Department of Ophthalmology; Provincial Public Hospital, São Paulo (HSPE), SP, Brazil

${ }^{3}$ Department of Ophthalmology; State University of São Paulo (USP), São Paulo, SP, Brazil

Correspondence: MLS Jordão, Department of Ophthalmology, Avenida Professor João Fiusa, 536, Ribeirão Preto, São Paulo, 14025-320, Brazil

Tel: +55163913 4758; Fax: + 551639134758 E-mail: jordao.marcelo@ terra.com.br

Received: 30 June 2008 Accepted in revised form: 15 August 2008 Published online: 12 September 2008

The authors have no proprietary interest in the material presented in this manuscript

\begin{abstract}
Aims To compare the IOP measurements obtained with dynamic contour tonometry (DCT) and Goldmann applanation tonometry (GAT), and to analyse the influence of central corneal thickness (CCT) and age on both measurements, and the influence of the quality score on DCT readings.

Methods A total of 500 healthy subjects with no prior history of glaucoma or ocular hypertension (age: 7-86 years) were consecutively recruited. GAT, DCT, and CCT measurements were obtained from both eyes of each individual, in this order, by three observers. The mean of five CCT measurements was used for analysis. DCT measurements were accepted when quality scores varied between 1 (higher quality) and 3 (lower quality).

Results Mean DCT measurements were $3.2 \mathrm{mmHg}$ higher than GAT readings. CCT values varied between 449 and $653 \mu \mathrm{m}$. IOP measured by GAT correlated strongly with CCT $\left(r^{2}=0.28, P=<0.001\right)$, whereas DCT readings correlated poorly with CCT $\left(r^{2}=0.01\right.$, $P=0.017)$. Both DCT $\left(r^{2}=<0.01, P=0.044\right)$ and GAT $\left(r^{2}=0.01, P=<0.001\right)$ measurements correlated poorly with age. Bland-Altmann analysis revealed disagreement between DCT and GAT readings, with $95 \%$ confidence intervals of $\pm 6.7 \mathrm{mmHg}$. Quality scores for DCT measurements were $1(n=369,36.9 \%)$, $2(n=340,34.0 \%)$, and $3(n=291,29.1 \%)$. DCT readings with quality score of 3 $(18.8 \pm 3.4 \mathrm{mmHg})$ were significantly higher than those with quality scores of 1 $(16.7 \pm 2.9 \mathrm{mmHg})$ and $2(17.4 \pm 2.9 \mathrm{mmHg}$; $P=<0.001$ )

Conclusions DCT is not influenced by CCT, unlike GAT. Both DCT and GAT measurements are not influenced by age. DCT
\end{abstract}

MLS Jordão, ${ }^{1,2}$, A Pedroso de Carvalho Lupinacci' EL Ferreira', IJF Enomoto ${ }^{1}$ and VP Costa ${ }^{1,3}$

measurements with lower quality scores are associated with higher readings.

Eye (2009) 23, 1364-1369; doi:10.1038/eye.2008.278; published online 12 September 2008

Keywords: glaucoma; dynamic contour tonometry; Pascal; Goldmann; pachimetry; age

\section{Introduction}

Intraocular pressure (IOP) is a key component to the diagnosis and treatment of glaucoma, and Goldmann applanation tonometry (GAT; model R900, Haag-Streit, Koeniz, Switzerland) remains the gold standard for this measurement. There are, however, known sources of error that influence the measurements obtained with this method, including corneal thickness and curvature. ${ }^{1-3}$ IOP measured with GAT may be overestimated in ocular hypertensive individuals because of commonly observed thicker $\mathrm{CCT}_{1}{ }^{4-6}$ whereas normal tension glaucoma is reported to be associated with CCTs in the low $500-\mu \mathrm{m}$ range. $^{7}$

Applanation tonometry is based on the Imbert-Fick Law, which states that, in a thin, dry and perfectly elastic sphere, the force applied to flatten the sphere has the same intensity of the force promoted by internal pressure. However, the human cornea is neither uniformly elastic, nor thin, nor dry.,

In 2005, dynamic contour tonometry (DCT) was developed as a method of tonometry that is theoretically affected neither by CCT nor by corneal curvature. ${ }^{10-13}$ DCT is based on a different physical principle: when the contours of the corneal surface and the tonometer match, the pressure measured at the surface of the eye is supposed to equal the pressure inside the 
eye. ${ }^{14}$ DCT provides a score $(Q)$ representing the quality of the IOP measurement, that ranges from 1 (optimum) to 5 (unacceptable). ${ }^{3,15}$ In studies performed on human cadaver eyes, IOP values obtained by DCT were significantly closer to the manometric reference pressure than those obtained using GAT. ${ }^{11}$ Furthermore, IOP values determined by GAT before and after laser in situ keratomileusis (Lasik) were significantly different, whereas pre- and postoperative DCT values were found to be nearly the same. ${ }^{13}$

The purpose of this study was to compare the IOP measurements obtained with DCT and GAT, and to analyse the influence of CCT and age on both measurements, and the influence of the quality score on DCT readings.

\section{Materials and methods}

This prospective cross-sectional study included 500 consecutive healthy subjects, recruited among relatives of patients at the Department of Ophthalmology of the State University of Campinas and the Provincial Public Hospital of São Paulo, Brazil. This study followed the tenets of the Declaration of Helsinki and was approved by the Institutional Review Board of the University of Campinas. Written informed consent was obtained from all participants.

Inclusion criteria were patients with open angles at gonioscopy, best-corrected visual acuity of 20/40 or better OU, no history of increased IOPs, and normal optic discs, as evaluated by a clinical expert. Optic discs were considered normal in the absence of the following findings: oedema, pallor, $\mathrm{C} / \mathrm{D}$ ratio $>0.5$, localized rim loss, disc haemorrhage, or $\mathrm{C} / \mathrm{D}$ ratio asymmetry $>0.2$.

Exclusion criteria were previous intraocular surgery, refractive surgery, conjunctival or intraocular inflammation, corneal abnormalities (such as oedema or scars), glaucoma, family history of glaucoma, and history of ocular trauma.

All subjects underwent an ophthalmological examination including best-corrected visual acuity evaluation, slit-lamp examination, gonioscopy, and fundus biomicroscopy with a 90-D lens. Both eyes were selected for analysis. Central corneal thickness (CCT) was measured with ultrasonic pachimetry (Micropach 200P +, Sonomed, Lake Success, NY, USA). The pachimetry probe was placed on the centre of the cornea and the mean of five readings was used for the analysis.

The GAT was calibrated according to the manufacturer's guidelines and used at the slit lamp. The Pascal DCT instrument (SMT Swiss Microtechnology AG, Zurich, Switzerland) is a device mounted on the slit lamp and is self-calibrating. It consists of a sensor tip with a 10.5-mm radius of curvature, a concave surface, and a miniaturized pressure sensor (1.2-mm diameter) integrated into the centre of the contact surface. A beeping sound is emitted by the device when the tip is in contact with the cornea and correctly positioned. ${ }^{10}$ The IOP measurement takes approximately 75 seconds. Only measurements with a $Q$-value $\leqslant 3$ were accepted.

All measurements with GAT and DCT were taken by one of the three experienced examiners. Three readings were taken with each instrument and the mean was used for analysis. The IOP measurements were always taken first with the Goldmann tonometer, followed by DCT and CCT, after a minimum interval of $10 \mathrm{~min}$ between measurements.

Intermethod agreement between the tonometers was assessed using the method devised by Bland and Altman. ${ }^{15}$ Mean difference and 95\% limits of agreement were calculated. Linear regression analysis was used to analyse the influence of CCT and patient age on GAT and DCT readings. To further analyse the influence of CCT on readings obtained with both instruments, we divided the eyes in five categories according to CCT - group 1: $\mathrm{CCT} \leqslant 500 \mu \mathrm{m}$; group 2: CCT from 501 to $540 \mu \mathrm{m}$; group 3: CCT from 541 to $560 \mu \mathrm{m}$; group 4: CCT from 561 to $600 \mu \mathrm{m}$; and group 5: CCT $>600 \mu \mathrm{m}$. Measurements obtained with GAT and DCT were compared in each category using ANOVA.

To evaluate the influence of the quality of the measurement obtained with DCT, mean values of readings with a quality score of 1 were compared to mean values of measurements with quality scores 2 and 3 using ANOVA. Statistical analysis was performed using SPSS Version 11.0 (SPSS Inc., Chicago, IL, USA). $P$-values of less than 0.05 were considered statistically significant.

\section{Results}

Among the 500 healthy subjects (1000 eyes) included in this study, the mean age was $46.8 \pm 16.4$ years (range: 7-86 years), $324(64.8 \%)$ were female, 170 (34.0\%) were African-American, 323 (64.6\%) were Caucasian, and $7(1.4 \%)$ were Asian (patients self-defined their race).

Mean IOP measured by GAT $(14.3 \pm 3.7 \mathrm{mmHg})$ was significantly lower than the mean IOP measured by DCT $(17.2 \pm 3.2 \mathrm{mmHg}), P=<0.001$. There was a strong correlation between GAT and DCT measurements $\left(r^{2}=0.26, P=<0.001\right)$, Figure 1. However, the BlandAltmann analysis revealed disagreement between DCT and GAT readings, with $95 \%$ confidence intervals of $\pm 7.0 \mathrm{mmHg}$, Figure 2 .

Mean CCT was 543.6 $\pm 35.4 \mu \mathrm{m}$ (range: $449-653 \mu \mathrm{m}$ ). IOP measured by GAT correlated strongly with CCT $\left(r^{2}=0.28, P=<0.001\right)$, whereas DCT readings correlated 


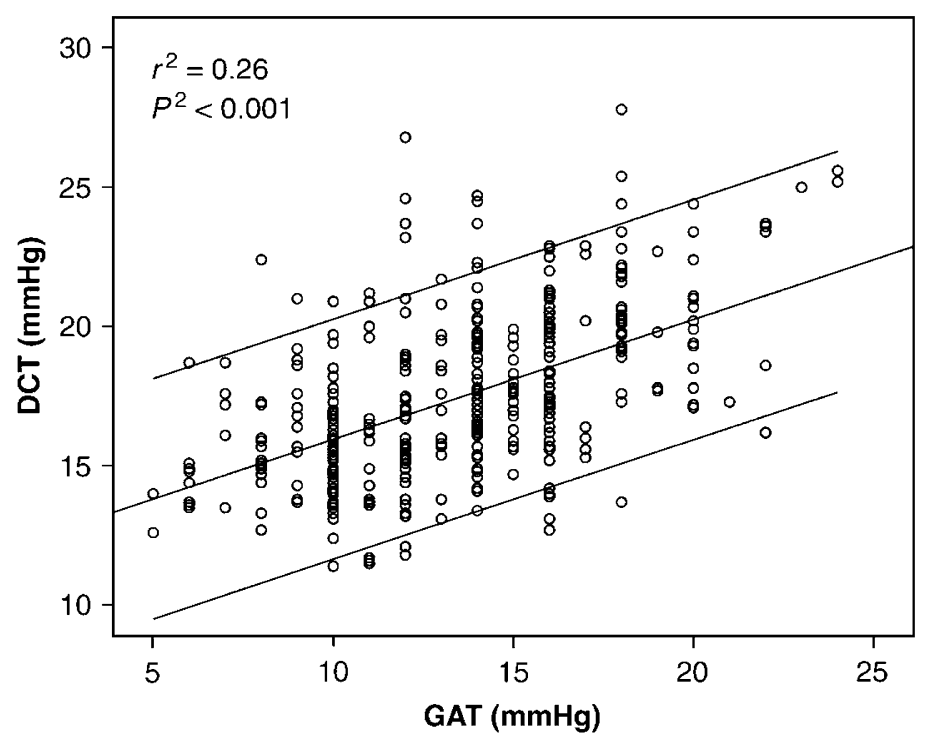

Figure 1 Scatter plot showing DCT measurements compared with GAT measurements in 500 healthy individuals. GAT = Goldmann aplanation tonometry; DCT $=$ dynamic contour tonometry.

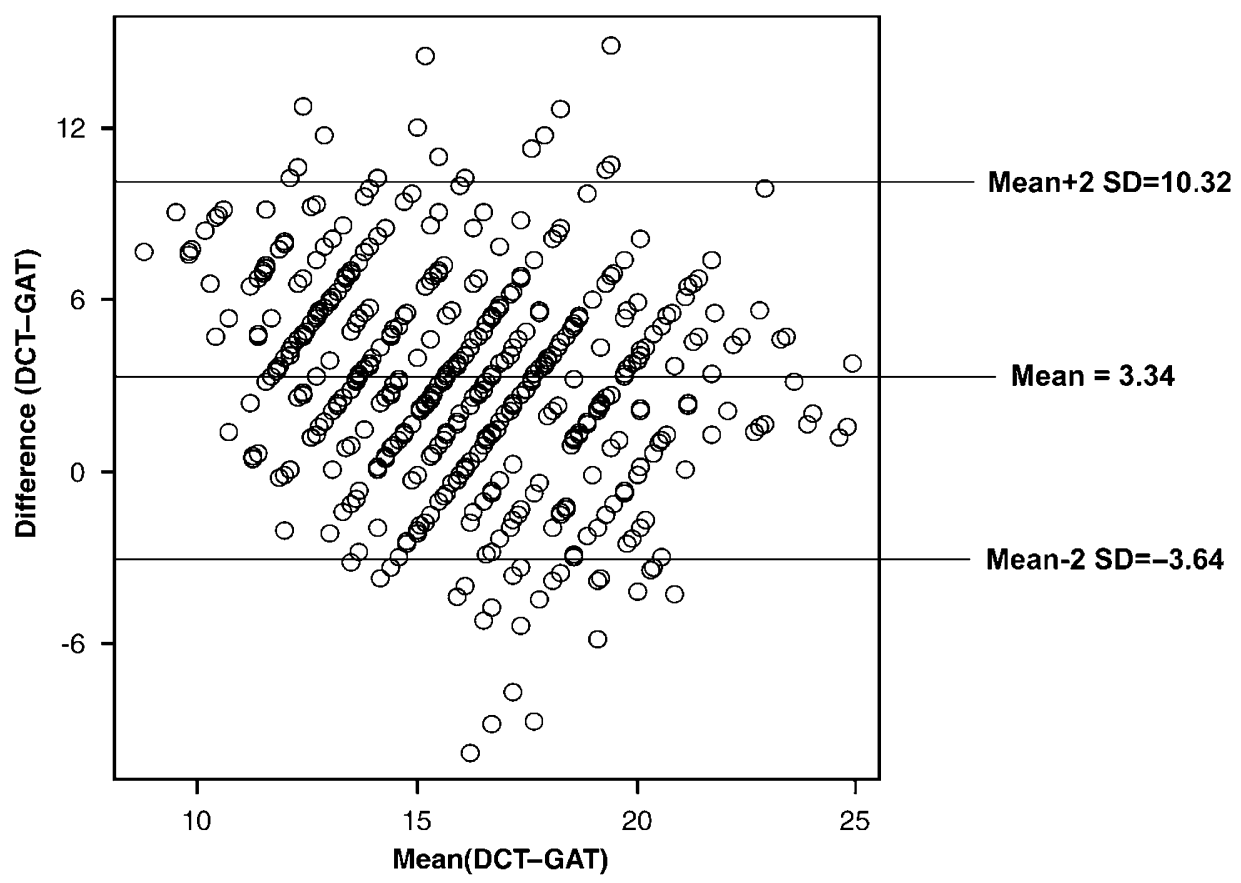

Figure 2 Bland-Altman plot of the difference between DCT and GAT readings (measurements in $\mathrm{mmHg}$ ).

poorly with CCT $\left(r^{2}=0.01, P=0.017\right)$. After CCT stratification into five categories, mean DCT IOP was significantly higher than GAT $(P=<0.001)$ in groups $1-4$, but was significantly lower than GAT in group 5 $(P=<0.001)$. Table 1. Both DCT $\left(r^{2}=<0.01, P=0.044\right)$ and GAT $\left(r^{2}=0.01, P=<0.035\right)$ measurements correlated poorly with age.
Quality scores for DCT measurements were classified as $1(n=369,36.9 \%), 2(n=340,34.0 \%)$, and $3(n=291$, $29.1 \%$ ). Mean IOP readings by DCT with a quality score of $3(18.8 \pm 3.3 \mathrm{mmHg})$ were significantly higher than those with quality scores of $1(16.6 \pm 2.9 \mathrm{mmHg})$ and 2 $(17.4 \pm 2.9 \mathrm{mmHg}), P=<0.001$. There were no significant differences between mean age, mean CCT, and the 
Table 1 Mean DCT readings and mean GAT measurements according to CCT stratification

\begin{tabular}{lccccc}
\hline & $C C T \leqslant 500 \mu m$ & $501 \leqslant C C T \leqslant 540 \mu m$ & $541 \leqslant C C T \leqslant 560 \mu m$ & $561 \leqslant C C T \leqslant 600 \mu m$ & $C C T>600 \mu m$ \\
\hline DCT (mmHg) & $16.7 \pm 3.5$ & $17.5 \pm 3.0$ & $17.47 \pm 3.0$ & $18.07 \pm 3.0$ & $17.32 \pm 3.0$ \\
GAT (mmHg) & $11.2 \pm 2.7$ & $13.18 \pm 3.2$ & $14.10 \pm 2.9$ & $16.30 \pm 3.3$ & $19.49 \pm 2.3$ \\
$\Delta$ DCT $/$ GAT & 5.47 & 4.30 & 3.37 & 1.77 & -2.17 \\
$P$ & $P<0.001$ & $P<0.001$ & $P<0.001$ & $P<0.001$ & $P<0.001$ \\
\hline
\end{tabular}

$\mathrm{CCT}=$ central corneal thickness; $\mathrm{DCT}=$ dynamic contour tonometry; GAT = Goldmann aplanation tonometry.

Table 2 Mean DCT, mean age, mean CCT, gender, and race distribution according to the quality score $(Q)$

\begin{tabular}{lcccr}
\hline & $Q=1$ & $Q=2$ & $Q=3$ & \multicolumn{1}{c}{$P$} \\
\hline DCT (mmHg) & $16.6 \pm 2.9$ & $17.4 \pm 2.9$ & $18.8 \pm 3.3$ & $<0.001$ \\
Age (years) & $45.88 \pm 16.43$ & $46.79 \pm 16.16$ & $48.08 \pm 16.65$ & 0.231 \\
CCT ( $\mu \mathrm{m})$ & $543.9 \pm 36.6$ & $544.7 \pm 34.3$ & $541.9 \pm 35.7$ & 0.603 \\
Gender (M:F) & $131: 238$ & $115: 225$ & $106: 185$ & 0.784 \\
Race & $247: 117: 5$ & $213: 120: 7$ & $186: 103: 2$ & 0.429 \\
(C:AA:O) & & & & \\
\hline
\end{tabular}

$\mathrm{A}=$ Asian $; \mathrm{AA}=$ African-American $\mathrm{C}=$ Caucasian $\mathrm{CCT}=$ central corneal thickness; $\mathrm{DCT}=$ dynamic contour tonometry; $\mathrm{M}=$ male.

gender distribution among eyes with quality scores of 1,2 , and 3. Table 2 .

\section{Discussion}

There is increasing interest in developing alternative, clinically applicable tonometers that eliminate or minimize the effect of CCT when measuring IOP, especially in structurally normal thick or thin corneas and in eyes following refractive surgery.

In our series including 500 individuals, DCT measurements were a mean of $3.2 \mathrm{mmHg}$ higher than GAT readings. This value appears greater than those reported previously, which varied between 0.94 and 2 mmHg. ${ }^{11,12,16,17}$ The tendency of DCT to give higher IOP readings compared with GAT is in good agreement with the results of a recently published study, which demonstrated that the IOP measured by applanation tonometry was $1.2-2 \mathrm{mmHg}$ lower than the IOP measured manometrically in human in vivo eyes. ${ }^{18}$ The higher readings obtained with DCT may be expected, as DCT was calibrated against a manometrically controlled standard pressure rather than a GAT pressure reading. ${ }^{18}$

Similarly to other studies, ${ }^{13,19}$ we found a strong correlation between GAT and DCT IOP measurements, although the agreement between the measurements were poor according to the Bland-Altmann analysis. In fact, this discrepancy is not surprising, as it is not uncommon to observe good correlation and poor agreement between different instruments measuring the same parameter. ${ }^{15}$ When we evaluate correlation, we assess the relationship between measurements. We expect a good correlation between GAT and DCT IOP measurements, as both instruments are measuring the same parameter. When agreement is analysed, we check whether the instruments are giving the same results, which was not the case when DCT and GAT were compared. The lack of agreement between GAT and DCT was also expected, as GAT is influenced by CCT, and DCT is not.

In their meta-analysis, Doughty and Zaman ${ }^{19}$ found a chronological upward trend in the reported averages for CCT during a 30-year period that is thought to be due to the change from optical to ultrasonic measuring methods. The group-average value for CCT using optical pachymetry was $525 \mu \mathrm{m}$ (median), compared to $544 \mu \mathrm{m}$ (median) for ultrasonic pachymetry, which is consistent with our finding of $543 \mu \mathrm{m} .^{20-24}$

In our series, GAT showed a significant correlation with CCT, contrary to DCT. In accordance with other studies, ${ }^{6,11,17,25}$ we observed that CCT affects GAT IOP measurements. We also demonstrated that GAT measurements are lower than DCT readings in corneas with CCTs $<600 \mu \mathrm{m}$, but they become higher in thick corneas (CCT $>600 \mu \mathrm{m})$. It is expected that the mean measured IOP difference (DCT minus GAT) should, in theory, become negative in thicker corneas as GAT tends to overestimate IOP in these eyes. ${ }^{6}$ In agreement with our findings, $\mathrm{Ku}$ et $a l^{17}$ observed that Pascal DCT tended to give higher readings than GAT in thinner and normal corneas, whereas a trend for GAT measurements to be greater than those taken with Pascal DCT was noted in thicker corneas.

As previously shown by Salvetat et al, ${ }^{16}$ our study showed that neither DCT nor GAT IOP readings were correlated with age. Interestingly, Kotecha et $a l^{26}$ found that GAT-DCT differences were positively associated with age. In younger eyes, DCT readings were greater than GAT readings, but this difference reversed in older eyes. It has been suggested that age-related increase in corneal 'stiffness' may induce a further measurement error with GAT. ${ }^{27}$ DCT measurements may be less affected by this change in corneal biomechanics.

In this study, only DCT measurements with a $Q$-value $\leqslant 3$ were accepted. For the first time to our knowledge, the influence of quality scores on DCT measurements 
was evaluated. Unfortunately, the manufacturer does not provide the exact algorithms involved in the calculation of the quality score or the reason for rejecting a specific IOP measurement. However, the quality score may give important information about the reliability of the measurement, which may represent an advantage of DCT over GAT. In GAT, there are several sources of error, which are not automatically evaluated by the instrument, including hypo- or hyperfluorescence of the cornea-too wide, too small, or vertically misaligned mires; or prolonged corneal contact resulting in false too low or false too high IOP estimates. ${ }^{28}$ We have demonstrated that DCT readings with poor quality $(Q=3)$ may be significantly greater than better quality measurements $(\mathrm{Q}=1$ or 2$)$. This finding was not explained by differences in CCT, age, race, or gender distribution (Table 2) and indicates that DCT results are influenced by the quality of the measurement.

In conclusion, this study has demonstrated that DCT measurements are not influenced by age or CCT, and tend to be higher than GAT readings in corneas with CCTs up to $600 \mu \mathrm{m}$. Furthermore, DCT readings with quality scores of 3 or 2 tend to be higher than better quality measurements. We suggest that DCT readings with quality scores of 3 or more should be discarded, and that the examiner should aim for a measurement with quality scores of 1 .

\section{References}

1 Glaucoma Primário de Ângulo Aberto. I Consenso da Sociedade Brasileira de Glaucoma. BG Editora: Sao Paulo, 2001.

2 Weinreb RN, Khaw PT. Primary open-angle glaucoma. Lancet 2004; 363: 1711-1720.

3 Liu J, Roberts CJ. Influence of corneal biomechanical properties on intraocular pressure measurement: quantitative analysis. J Cataract Refract Surg 2005; 31: 146-155.

4 Heijl A, Leske MC, Bengtsson B, Hyman L, Hussein M. Reduction of intraocular pressure and glaucoma progression: results from the Early Manifest Glaucoma Trial. Arch Ophthalmol 2002; 120: 1268-1279.

5 Kass MA, Heuer DK, Higginbotham EJ, Johnson CA, Keltner JL, Miller JP et al. The Ocular Hypertension Treatment Study: a randomized trial determines that topical ocular hypotensive medication delays or prevents the onset of primary open-angle glaucoma. Arch Ophthalmol 2002; 120: 701-713; discussion 829-30.

6 Brandt JD, Beiser JA, Kass MA, Gordon MO. Central corneal thickness in the Ocular Hypertension Treatment Study (OHTS). Ophthalmology 2001; 108: 1779-1788.

7 Morad Y, Sharon E, Hefetz L, Nemet P. Corneal thickness and curvature in normal-tension glaucoma. Am J Ophthalmol 1998; 125: 164-168.

8 Goldmann H, Schmidt T. [Applanation tonometry.]. Ophthalmologica 1957; 134(4): 221-242.
9 Allingham RR, Damji KF, Freedman S, Moroi SE, Shafranov G, Shields MB. Shield's Textbook of Glaucoma, 5a ed. Lippincott Williams \& Wilkins, 2004.

10 Kanngiesser HE, Kniestedt C, Robert YC. Dynamic contour tonometry: presentation of a new tonometer. J Glaucoma 2005; 14: 344-350.

11 Doyle A, Lachkar Y. Comparison of dynamic contour tonometry with goldman applanation tonometry over a wide range of central corneal thickness. J Glaucoma 2005; 14: 288-292.

12 Kaufmann C, Bachmann LM, Thiel MA. Comparison of dynamic contour tonometry with goldmann applanation tonometry. Invest Ophthalmol Vis Sci 2004; 45: 3118-3121.

13 Kniestedt C, Lin S, Choe J, Bostrom A, Nee M, Stamper RL. Clinical comparison of contour and applanation tonometry and their relationship to pachymetry. Arch Ophthalmol 2005; 123: 1532-1537.

14 Punjabi OS, Ho HK, Kniestedt C, Bostrom AG, Stamper RL, Lin SC. Intraocular pressure and ocular pulse amplitude comparisons in different types of glaucoma using dynamic contour tonometry. Curr Eye Res 2006; 31(10): 851-862.

15 Bland J, Altman D. Statistical methods for assessing agreement between two methods of clinical measurement. Lancet 1986; 1: 307-310.

16 Salvetat ML, Zeppieri M, Tosoni C, Brusini P. Comparisons between Pascal dynamic contour tonometry, the TonoPen, and Goldmann applanation tonometry in patients with glaucoma. Acta Ophthalmol Scand 2007; 85: 272-279.

17 Ku JY, Danesh-Meyer HV, Craig JP, Gamble GD, McGhee $\mathrm{CN}$. Comparison of intraocular pressure measured by Pascal dynamic contour tonometry and Goldmann applanation tonometry. Eye 2006; 20: 191-198.

18 Kniestedt C, Nee M, Stamper RL. Dynamic contour tonometry: a comparative study on human cadaver eyes. Arch Ophthalmol 2004; 122: 1287-1293.

19 Doughty MJ, Zaman ML. Human corneal thickness and its impact on intraocular pressure measures: a review and meta-analysis approach. Surv Ophthalmol 2000; 44: 367-408.

20 Bhan A, Browning AC, Shah S, Hamilton R, Dave D, Dua HS. Effect of corneal thickness on intraocular pressure measurements with the pneumotonometer, Goldmann applanation tonometer, and Tono-Pen. Invest Ophthalmol Vis Sci 2002; 43: 1389-1392.

21 Gunvant P, Baskaran M, Vijaya L, Joseph IS, Watkins RJ, Nallapothula $\mathrm{M}$ et al. Effect of corneal parameters on measurements using the pulsatile ocular blood flow tonograph and Goldamann applanation tonometer. Br J Ophthalmol 2004; 88: 518-522.

22 Shah S, Chatterjee A, Mathai M, Kelly SP, Kwartz J, Henson D et al. Relationship between corneal thickness and measured intraocular pressure in general ophthalmology clinic. Ophthalmology 1999; 106: 2154-2160.

23 Bron AM, Creuzot-Gauchert C, Goudeau-Boutillon S, d'Athis P. Falsely elevated intraocular pressure due to increased central corneal thickness. Graefes Arch Clin Exp Ophthalmol 1999; 237: 220-224.

24 Wolfs RC, Klaver CC, Vingerling JR, Grobbee DE, Hofman A, de Jong PT. Distribution of central corneal thickness and its association with intraocular pressure: The Rotterdam Study. Am J Ophthalmol 1997; 123(6): 767-772.

25 Pache M, Wilmsmeyer S, Lautebach S, Funk J. Dynamic contour tonometry versus Goldmann applanation 
tonometry: a comparative study. Graefes Arch Clin Exp Ophtahlmol 2005; 243: 763-767.

26 Kotecha A, White ET, Shewry JM, Garway-Health DF. The relative effects of corneal thickness and age on Goldmann applanation tonometry and dynamic contour tonometry. Br J Ophthalmol 2005; 89: 1572-1575.
27 Friedenwald JS. Contribuition to the therapy and practice of tonometry. Am J Ophthalmol 1937; 20: 985-1024.

28 Whitacre MM, Stein R. Sources of error with use of Goldmann-type tonometers. Surv Ophthalmol 1993; 38: $1-30$. 